\title{
PROCESSO ENSINO-APRENDIZAGEM DA TÉCNICA DE PALPAÇÃO E MENSURAÇÃO UTERINA: influência da prática
}

\author{
Clarice Heiko Muramatsu \\ Ana Maria Kazue Miyadahira
}

MURAMATSU, C.H.; MIYADAHIRA, A.M.K. Processo ensino-aprendizagem da técnica de palpação e mensuração uterina: influência da prática. Rev.Esc.Enf.USP, v.30, n.3, p.45672, dez. 1996.

O presente estudo relacionado ao processo ensino-aprendizagem da técnica de palpação e mensuração uterina, teve como objetivos, analisar a influência da prática em relação à habilidade e analisar o conhecimento adquirido da técnica dos estudantes de Graduação em Enfermagem. Aplicando o "modelo padrão" elaborado para este estudo, os estudantes foram avaliados em 2 etapas; os resultados obtidos permitiram concluir pela importância da prática no processo ensino-aprendizagem de uma habilidade perceptivo-motora, verificada através da melhora do desempenho dos estudantes em relação à habilidade e ao conhecimento adquirido.

UNITERMOS: Habilidade perceptivo-motora. Palpação e mensuração da altura uterina

\section{1 - INTRODUÇÃo}

O processo de ensino-aprendizagem, em Enfermagem, possui características peculiares, por ser eminentemente tébrico-prático. Apresenta ainda particularidades devido à necessidade de se desenvolver uma gama variada de procedimentos que envolvem habilidades perceptivo-motoras para a prestação do cuidado de enfermagem.

MAGILL (1984 a) define habilidade como uma atividade ou tarefa que precisa ser aprendida para ser executada correta e eficientemente e ressalta que as pessoas diferem na capacidade de aprender e executar habilidades motoras.

\footnotetext{
* Extraída da Dissertação de Mestrado apresentada à Escola de Enfermagem da USP.

** Mestre em Enfermagem. Professor Assistente da Faculdade de Enfermagem do Hospital Israelita Albert Einsten

*** Professor Doutor do Departamento de Enfermagem Médico-Cirúrgico da Escola de Enfermagem da USP
} 
Vários estudos demonstram a preocupação dos docentes em como processar o ensino destas habilidades. FRIEDLANDER (1984), GOMEZ ; GOMEZ (1984), BACHMAN (1990), MIYADAHIRA (1990), OERMANN (1990), DARIDO (1991), KIMURA (1991), MELO (1993), MIYADAHIRA; KOIZUMI (1993) enfatizam que o ensino de habilidades motoras deve ser realizado de maneira gradativa para facilitar a aprendizagem e que a prática delas é fundamental para o aprendizado, pois permite refinar ou diversificar a execução dos procedimentos.

A aprendizagem de uma habilidade perceptivo-motora, como a técnica de palpação e mensuração uterina (TPMU), necessita que o professor, neste processo, organize sistematicamente uma série gradual de situações, fornecendo informações ao estudante para a execução dos procedimentos específicos da disciplina Enfermagem Obstétrica, e proporcione experiências ricas, cuja qualidade e freqüencia assegurem o desempenho desta habilidade.

Para OERMANN (1990), habilidades psicomotoras são partes integrantes da prática de enfermagem e são utilizadas, tanto na avaliação física do cliente, como na implementação do cuidado.

Para CRANE (1977), a avaliação física do paciente assegura a consistência dos dados levantados, melhora a qualidade e a quantidade de informações que se pode obter do paciente, estabelece, desde o início, uma relação interpessoal com o paciente e possibilita uma assistência individualizada.

Assistência de enfermagem sistematizada e individualizada segundo KIMURA (1991), baseia-se na identificação dos problemas, através de uma avaliação física criteriosa, com base no exame físico.

Acredita-se que o exame físico seja uma atividade imprescindivel para a operacionalização de uma assistência sistematizada de enfermagem de boa qualidade. Para tanto, cabe as escolas de Enfermagem capacitarem os futuros profissionais para a execução não somente da referida atividade, como também para a consulta e a prescrição da assistência de enfermagem, conforme a Lei 7.498 de 25/06/86 (BRASIL, 1986) que dispõe sobre a regulamentação do exercício da enfermagem.

Em se tratando do exame físico em gestante e puérpera é incontestável a importância da palpação e mensuração uterina, como parâmetro de avaliação no ciclo gravídico puerperal. Estudos realizados por BELIZAN et al (1978), ENG̈STROM; WORK (1992), ENGSTROM et al (1993), ENGSTROM; MCFARLIN; SAMPSON (1993), ENGSTROM; SITTLER; SWIFT (1994), NEME (1994 a) afirmam que a mensuração da altura uterina permite a identificação de distúrbios do crescimento fetal, gestação múltipla, distúrbio na produção de líquido amniótico, mola hidatiforme e erros na idade gestacional. Acrescentam que a técnica de mensuração da altura uterina deva ser utilizada largamente em clínicas por ser um método inócuo, acessível, barato, não invasivo e aceito pela cliente.

HAWKINS; GORVINE (1985), JENSEN; BOBAK (1985 b), DELASCIO; GUARIENTO (1987), REEDER; MARTIN (1987), FARRER (1990) mencionam 
outros aspectos na mensuração da altura uterina, como o acompanhamento da involução do útero no puerpério, e recomendam que a avaliação deva ser diária, uma vez que o tamanho do útero involui de 1 a 2 centímetros por dia e, em torno do décimo dia, se torna um 6́rgão intrapélvico. NEME (1994 b) alerta para a tríade da endometrite puerperal que se caracteriza por subinvolução uterina, dor à palpação e amolecimento corporal (Tríade de Bumm).

Segundo ARAÚJ(); MARETTI (1991) as etapas do exame físico obstétrico são inspeção, palpação, mensuração, ausculta e toque.

A mensuração da altura uterina é uma das etapas da propedêutica obstétrica amplamente utilizada na prática clínica pelas enfermeiras e médicos obstetras na avaliação de gestantes e puérperas. Historicamente a mensuração da altura uterina começou a ser utilizada no século XVIII, quando a semiologia obstétrica dependia totalmente da avaliação clínica.

Em 1871, Ahlfeld citado por EN(ASTROM; SITTLER (1993) foi o primeiro investigador a estudar a mensuração da altura do útero através do pelvímetro. Porém, o uso desta técnica para avaliação da gravidez foi limitada na época, por ser um procedimento invasivo, causando desconforto e risco de infecção à gestante, uma vez que a extremidade do pelvímetro era introduzida na vagina contra a cabeça fetal. Posteriormente, com objetivo de minimizar os efeitos e difundir este procedimento, adotou-se como limite inferior a borda superior da sínfise púbica.

A mensuração da altura do útero, através da fita métrica, foi descrita por Spiegelberg citado por ENGSTROM; SITTLER (1993) que preconizava como limite inferior a identificação da parte superior da sínfise púbica e, quanto ao fundo uterino recomendava-se que fosse efetuada a percussão.

Mcdonald popularizou a técnica da mensuração com a fita métrica em duas publicações, de 1906 e 1910. A partir disso, surgiram várias técnicas que apresentavam variações quanto à posição da gestante, as condições da bexiga, o contato da fita com o abdome materno e outros.

Para NEME (1994 a), a técnica consiste em fixar a fita métrica no meio da borda superior da sínfise púbica, deslizá-la sobre a superfície mediana da parede abdominal, com a borda cubital da mão, até o encontro do fundo uterino .

BELIZAN et al (1978), WORTHEN; BUSTILLO (1980), JENSEN; BOBAK (1985 a), ENGSTROM; WORK (1992), ENGSTROM; SITTLER (1993), ENGSTROM et al (1993), ENGSTROM; SITTLER; SWIFT (1994) acrescentam que para a obtenção da mensuração correta e confiável se faz necessário a elaboração de um protocolo que sirva de guia para a execução da técnica em questão pelos profissionais, ressaltando a posição materna durante a mensuração, as condições da bexiga, presença ou ausência das contrações uterinas, limites da sínfise púbica e do fundo uterino e o contato da fita métrica com a pele da paciente.

Pela importância e especificidade, deve haver preocupação por parte dos docentes com o ensino dos procedimentos especificos desta área, geralmente a serem desenvolvidos na disciplina de Enfermagem Obstétrica, no Curso de Graduação em Enfermagem. 
Portanto, torna-se primordial capacitar os futuros profissionais, proporcionando-lhes oportunidades para que desenvolvam habilidades pertinentes à obstetricia, para melhor assistir a gestante, o recem-nascido e a puérpera.

Diante do que foi exposto questiona-se: a disciplina prepara o estudante para assistir a gestante e a puerpera quanto ao exame físico obstétrico? A prática acarreta modificaçōes em relação à habilidade em executar a TPMU? O conhecimento adquirido por estes estudantes quanto à técnica é igual ao inicialmente ensinado?

Diante do exposto, julgamos oportuno. neste estudo, avaliar o desempenho dos estudantes de Graduação em Enfermagem, quanto à habilidade e ao conhecimento adquiridos da TPMU. tendo como objetivos:

Analisar a influência da prática em relação à habilidade em executar a técnica de palpação o mensuração uterina, pelos estudantes de Graduação em Enfermagem.

Analisar a influência da prálica em relação ao conhecimento adquirido da técnica de palpação e mensuração uterina, pelos estudantes de Graduação em Enfermagem.

\section{2 - METODOLOGIA}

A população foi constituída por 35 estudantes do terceiro ano do Curso de Graduação em Enfermagem, de uma Instituição de Ensino privada, que cursavam a Disciplina de Enfermagem Obstétrica e Neonatal, sendo que os critérios para a inclusão neste estudo foram:

- Ter disponibilidade para participar do estudo e consentir na participação.

- Ter participado de todas as estratégias para o ensino desta técnica.

- Não possuir experiência anterior na técnica de palpação e mensuração uterina

Fizeram parte deste estudo puérperas de pos-parto normal e pós-parto fórceps. Foram excluídas as puérperas de pós-parto cesáreos para evitar desconforto: e para garantir a realização da técnica de acordo com o procedimento adotado neste estudo, foram ministradas pela pesquisadora, aula expositiva e demonstração dos passos sequênciais da técnica aos estudantes.

Para a coleta de dados foram elaborados os seguintes intrumentos: 
ANEXO I - Técnica de palpação e mensuração uterina - TPMU ( modelo padrão).

Para verificar o aprendizado dos estudantes em relação à técnica de palpação e mensuração uterina, elaborou-se um instrumento (ANEXO I), modelo padrão, sob a forma de escala de Likert, que foi utilizado como um "check-list" para comparar e avaliar a execução do procedimento antes e após a experiência prática. que corresponde à primeira e à segunda etapas do estudo. Este mesmo modelo padrão "check-list" foi utilizado para comparar e avaliar a descrição do procedimento realizado pelo estudante.

Este instrum ento forneceu os dados para avaliar a habilidade na execução da técnica.

\section{ANEXO II - Ficha para descrição da TPMU.}

Este instrumento consta de um espaço reservado para a descrição, pelos estudantes, dos passos da técnica de palpação e mensuração uterina, antes e após a prática no campo de estágio. Este instrumento forneceu os dados para avaliar o conhecimento adquirido do estudante.

ANEXO III - Criterios adotados para a avaliaşão da aprendizagem da TPMU.

Os critérios adotados para o preenchimento do instrumento, Anexo I, basearam-se na escala de Likert. Cada item da técnica foi classificado segundo as categorias $\mathrm{A}, \mathrm{B}, \mathrm{C}, \mathrm{D}$ ) e $\mathrm{E}$, às quais foi atribuído um peso (Anexo III). Optouse por atribuir A. quando o item preenchia por completo os critérios estabelecidos no modelo padrão. B quando o item não era executado ou executado incorretamente. (" quando na execução fosse omitido um dos passos do item. D quando fosse omitido dois passos do item e $\mathrm{E}$ quando fosse omitido três passos.

A coleta de dados ocorreu durante o estágio dos estudantes em um Hospital Público do Município de São Paulo. no período de abril a junho de 1994, em duas etapas.

A primeira etapa da coleta de dados ocorreu no primeiro dia de estágio, com os estudantes escalados para a unidade de puerpério. Após a seleção da puérpera, o estudante foi acompanhado individualmente na execução da TPMU, e os itens do "check-list" foram assinalados pela pesquisadora à medida que $o$ estudante executava a técnica, conforme definições estabelecidas no Anexo III.

Após a execução, foi entregue o Anexo II. onde os estudantes descreveram em itens os passos da TPMU em uma sala reservada da unidade. $\mathrm{Na}$ oportunidade, os estudantes foram orientados a realizar, durante o período de estágio, tantas palpações quantas fossem necessárias para o aprendizado da técnica em questão.

A 2a. etapa da coleta de dados ocorreu no último dia de estágio, nesta mesma unidade, seguindo os mesmos critérios utilizados na primeira etapa. $\mathrm{Na}$ ocasião os estudantes informaram à pesquisadora. do número de mensurações realizadas no período de estágio. 
Para a análise dos dados utilizou-se o teste t,-pareado, para comparação das notas ponderadas na 1a. e 2a. etapas do estudo, com nível de significância de $5 \%, \mathrm{t}=$ [ média diferença notas / ( desvio padrão da dif. / $\mathrm{n}$ ]. As notas correspondem às médias ponderadas dos 12 itens, que foram obtidas multiplicando o valor da pontuação do item pelo peso, segundo as categorias $\mathrm{A}$, $\mathrm{B}, \mathrm{C}, \mathrm{D}$ e $\mathrm{E}$ de cada item.

Se $|t|>(2,03 \%)$ valor crítico, pode-se concluir que houve indícios de melhora significativa do estudante na realização da TPMU; caso contrário, conclui-se que não houve indícios de aprendizagem.

\section{3 - RESULTADOS E COMENTÁRIOS}

A população deste estudo foi constituída de 35 estudantes do sexo feminino, a faixa etárea de 19 a 36 anos, com predominância entre 19 a 23 anos $(65,7 \%)$.

Segundo TANI et al (1988), SANCHES (1989) a aquisição, estabilização e diversificação das habilidades motoras básicas como andar, correr, pular e outros ocorrem aos 6.7 anos de idade e, aos 10.12 anos, se caracterizam fundamentalmente pelo refinamento e diversificação, alcançando o padrão maduro observado nos adultos. Portanto, supõe-se que os estudantes neste estudo tenham alcançado suficientemente o padrão maduro para o desenvolvimento de suas habilidades e para o aprendizado da TPMU.

Neste estudo foi estabelecido, como um dos critérios para participar, a ausência de experiência anterior na TPMU. MAGILL (1984 b) considera fundamental esta condição de excluir estudantes com diferentes graus de experiência, pois uma tarefa nova se constitui em um dos critérios para medir o desempenho de uma habilidade motora.

PASSOS (1989) define aprendizagem de uma habilidade motora nova quando o estudante realiza um movimento que ele ainda não realizou anteriormente, embora este movimento possa parecer semelhante a outros executados.

Embora a TPMU seja uma experiência nova para os estudantes e, portanto, uma habilidade a ser aprendida, não se pode deixar de citar que os mesmos tiveram oportunidades de desenvolver habilidades motoras semelhantes em outras disciplinas, quando da execução do exame físico onde se realiza a palpação de outros órgãos, facilitando desta forma o ensino e o aprendizado desta técnica através do princípio de transferência da aprendizagem .

Segundo MAGILL (1984 c), o estudante aprende esta nova experiência com mais facilidade, devido à influência de uma habilidade anteriormente praticada sobre esta nova; e, quanto mais alto for o grau de semelhança entre os componentes de suas habilidades, tanto maior será a quantidade de transferência. 
$O$ autor alerta para que este princípio da transferência da aprendizagem não seja negligenciado pelo professor, tanto no desenvolvimento do currículo como na metodologia de ensino, de forma que aquilo que é ensinado tenha mais possibilidade de ser transferido para novas situações de aprendizagem.

Com relação ao tempo utilizado na execução da técnica, podemos observar, no quadro 1, que a média do tempo utilizado na execução da TPMU, na la. e na 2a. etapas do estudo. foi respectivamente de 4,03 minutos e 5,11 minutos. A diferença entre as etapas foi de 1,08 minutos.

Quadro 1 - Media de tempo (min.) utilizado na execução da TPMU e o desvio padrão nas duas etapas. São Paulo, 1994.

\begin{tabular}{|l|c|c|}
\hline \multicolumn{1}{|c|}{ TEMPO } & 1a. etapa & 2a. etapa \\
\hline Média & 4.03 & 5.11 \\
Desvio padrão & 1.42 & 1.13 \\
\hline
\end{tabular}

MIYADAHIRA (1990), estudando a habilidade psicomotora da técnica de. injeção intramuscular. com 3 grupos de estudantes de enfermagem no laboratório e no campo clínico (hospital e unidade básica de saúde), observou que os estudantes que executaram a técnica no laboratório gastaram menos tempo. Liste fato pode estar relacionado com o tempo utilizado com os cuidados e orientações prestadas aos pacientess/clientes do hospital e unidade básica de saúde.

Para a realização da técnica, pressupõe-se que os estudantes executem todos os passos. Contudo, para esta diferença de 1.08 minutos pode ser justificado que na 1a. etapa, alguns dos passos da técnica não foram assimilados e, ao executar, eles os omitiram por completo ou parcialmente. Deve-se considerar ainda que a omissão dos passos pode estar relacionada a falta de interação com a puérpera e, desta forma, pode-se inferir que o estudante não havia incorporado o conhecimento quando realizou a técnica na la. etapa.

No entanto, desempenhar com exatidão a habilidade e executar rapidamente pode ser difícil no início da prática, como sugere OERMANN (1990), pois o estudante deve antes dominar os componentes individuais da habilidade, a seqüência a ser executada para melhorar o desempenho e a rapidez. A exatidão e a velocidade com os quais oprocedimento é executado variam consideravelmente de uma pessoa para outra.

Para se alcançar os objetivos fixados neste estudo, os estudantes foram orientados para que repetissem as mensurações ou vivenciassem estas experiências quantas vezes fossem necessárias para seu aprendizado.

Através do quadro 2 verificou-se que, em média, cada estudante realizou 16,8 mensurações. 
Quadro 2 - Número de mensurações realizadas na execução da TPMU. São Paulo, 1994.

\begin{tabular}{|c|c|}
\hline Ficha & No. de mensuraçסes \\
\hline 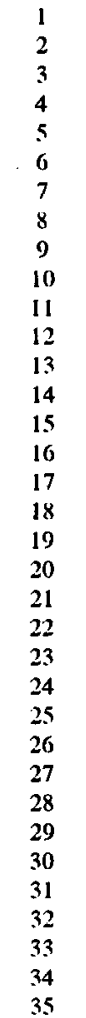 & $\begin{array}{l}16 \\
17 \\
16 \\
22 \\
15 \\
11 \\
18 \\
20 \\
20 \\
08 \\
15 \\
19 \\
30 \\
10 \\
15 \\
19 \\
25 \\
20 \\
29 \\
15 \\
18 \\
09 \\
16 \\
13 \\
22 \\
23 \\
19 \\
11 \\
09 \\
17 \\
12 \\
10 \\
20 \\
18 \\
14\end{array}$ \\
\hline
\end{tabular}

A falta de estudos nesta área dificultou o estabelecimento de uma relação de comparação com os resultados de outros autores. No entanto, esta média se torna significativa uma vez que na $2 a$. etapa (quadro 3 e 4), os erros cometidos diminuíram sensivelmente, permitindo inferir que a prática foi fundamental para o aprendizado da técnica. Porém, cabe ressaltar que a prática, o executar ou a simples atividade de repetir não constituem experiência se não estiverem acompanhadas da compreensão, por parte do estudante, das conseqüências do ato e de informações (feed-back) acerca de seu desempenho, para motivá-lo e orientá-lo.

KIMURA (1991). BER( $A M A S C O$ (1994) consideram a repetição um recurso pedagógico importante, principalmente em situação real de ensinoaprendizagem envolvendo habilidades perceptivo-motoras.

Segundo MIYADAHIRA, (1990) não basta transmitir a informação oralmente, ou somente por demonstração ou ainda por devolução única para a 
incorporação do conhecimento de uma atividade eminentemente de domínio motor: para a efetivação da aprendizagem. a prática ó obrigatória e essencial.

NOGUEIRA (1995) confirma que o estudante necessita de treino para que possa incorporar o procedimento, realizá-lo com segurança e executá-lo em menor tempo.

Quanto a análise global da habilidade ao executar (quadro 3) e ao conhecimento adquirido atraves da descricão (quadro 1), os quadros demonstram que a média de itens executados $(80.1 \%)$ e descritos $(81.1 \%)$ corretamente. foi maior na 2a. etapa do que na 1a. etapa. tendo como referência o modelo padrão. Este resultado indica que ocorreu melhora no desempenho do estudante da la. para a 2a. etapa do estudo, após um período de experiência prática. permitindo inferir que houve redução de erros observada através das mudanças de comportamento ocorridas no desempenho do estudante.

Quadro 3 Média e poreentagem de itens execulades eorretamente pelo estudante durante a execução da 'T'PMU. nas duas etapas do estudo. São Paulo. 1994.

\begin{tabular}{|c|c|c|}
\hline Etapa & Média (n=12) & $\%$ \\
\hline 1a. & 5.2 & 44.0 \\
2a. & 9.6 & 80.4 \\
\hline
\end{tabular}

Quadro 4 Média e porcentagem de itens citados corretamente, pelo estudante. na descrição da TPMU. nas duas etapas do estudo. São Paulo, 1994.

\begin{tabular}{|c|c|c|}
\hline Etapa & Média (n=12) & $\%$ \\
\hline 1a. & 5.6 & 47,6 \\
$2 \mathrm{a}$. & 9.7 & $\mathbf{8 1 , 4}$ \\
\hline
\end{tabular}

Segundo TANI (1989), a aquisição ou aprendizagem de habilidades motoras pode ser compreendida como um processo de eliminação gradativa de erros de "performance".

Observa-se, também. que o número médio de itens descritos foi maior que o número médio de itens executados, ou seja, os estudantes omitiram menos passos na descrição da técnica do que na execução. Este resultado pode ser justificado pela situação que envolve o estudan te nesto processo de aprendizagem. Durante a execução ele se encontra diante do professor e do paciente executando os passos e. neste momento. há dois latores que podem estar interferindo no resultado, o stress e a ansiedade. Na outra situação, ele se encontra em uma sala reservada, descrevendo a técnica longe dos fatores que poderiam estar desencadeando stress e ansiedade. 
Contudo, os resultados obtidos no estudo sobre a habilidade psicomotora da técnica de injeção intramuscular por MIYADAHIRA (1990) demonstraram que o número de itens executados foram maiores que o número de itens descritos, pois o estudante ao descrever, muitas vezes, deixou de citar todos os passos, por julgá-los irrelevantes ou implícitos. A autora alerta, em seu trabalho, para a ansiedade e o stress que podem ocorrer no início da experiência prática, e chama atenção para o processo de ensino-aprendizagem, especialmente da técnica da injeção intramuscular, a ansiedade gerada ao paciente/cliente, docente e ao estudante.

ANGELO (1989) confirma que na realização de um procedimento novo, o paciente e o campo de estágio são situações desconhecidas que tornam o estudante inseguro e assustado, dificultando o seu desempenho.

Em estudo realizado sobre o ensino do exame físico das mamas, BERGAMASCO (1994) constatou que a presença do professor e a dificuldade de interação com a paciente foram fatores que interferiram na execução dos procedimentos pelos estudantes.

O stress e a ansiedade, para OERMANN (1990), são fatores que influenciam o resultado da aprendizagem, pois freqüentemente as habilidades são ensinadas e praticadas nas enfermarias, deixando os erros cometidos à mostra aos professores, cliente/paciente e outros.

ROGERS (1976 a), KUSHNIR (1986), AMMON- GABERSON (1987) ressaltam que a ansiedade e ostress interferem na aprendizagem do adulto que por não gostar de se expor em público utiliza de subterfúgios para disfarçar a ansiedade e o stress, através de pensamentos confusos, rubor de face, olhares preocupados, retração, inabilidade de ouvir, confusão. Os autores chamam atenção sobre a importância de se reduzir as fatores geradores de ansiedade e stress.

MAGILL (1984 d) sugere ao professor que acalme o tom de voz, dê assistência individual e assegure ao estudante uma certa confiança em suas habilidades para possibilitar a redução destes sentimentos.

Com objetivo de verificar se ocorreu aprendizagem, após um periodo de treinamento, MAGILL ( 1984 b) recomenda que se aplique um teste de retenção, no primeiro dia e após um período de treinamento, se ocorrer diferença positiva neste escore pode se afirmar que houve aprendizagem. $O$ teste utilizado foi o teste t-pareado, que se obtém calculando-se as notas ponderadas obtidas pelos estudantes nas duas etapas do estudo. 
Quadro 5 - Distribuição das notas ponderadas e a diferença de notas obtidas, em relação à habilidade de executar a TPMU, durante as duas etapas do estudo. São Paulo, 1994.

\begin{tabular}{|c|c|c|c|}
\hline Ficha & $\begin{array}{c}\text { Nota ponderada } \\
\text { 1a. etapa }\end{array}$ & $\begin{array}{l}\text { Nota ponderada } \\
\text { 2a. etapa }\end{array}$ & Diferença de nota Execução \\
\hline $\begin{array}{c}1 \\
2 \\
3 \\
4 \\
5 \\
6 \\
7 \\
8 \\
9 \\
10 \\
11 \\
12 \\
13 \\
14 \\
15 \\
16 \\
17 \\
18 \\
19 \\
20 \\
21 \\
22 \\
23 \\
24 \\
25 \\
26 \\
27 \\
28 \\
29 \\
30 \\
31 \\
32 \\
33 \\
34 \\
35\end{array}$ & $\begin{array}{l}0,37 \\
0,64 \\
0,62 \\
0,84 \\
0,37 \\
0,36 \\
0,44 \\
0,41 \\
0,36 \\
0,17 \\
0,87 \\
0,93 \\
0,59 \\
0,60 \\
0,35 \\
0,66 \\
0,90 \\
0,31 \\
0,80 \\
0,38 \\
0,56 \\
0,51 \\
0,54 \\
0,36 \\
0,25 \\
0,35 \\
0,05 \\
0,47 \\
0,60 \\
0,35 \\
0,44 \\
0,79 \\
0,82 \\
0,50 \\
0,71\end{array}$ & $\begin{array}{l}0,79 \\
0,79 \\
0,77 \\
0,88 \\
0,88 \\
0,88 \\
0,79 \\
0,81 \\
0,93 \\
0,55 \\
0,72 \\
1,00 \\
0,88 \\
0,64 \\
1,00 \\
1,00 \\
1,00 \\
0,88 \\
0,74 \\
0,64 \\
0,91 \\
0,87 \\
0,90 \\
0,73 \\
0,87 \\
0,91 \\
0,87 \\
0,90 \\
0,64 \\
0,97 \\
0,84 \\
1,00 \\
1,00 \\
0,79 \\
0,87\end{array}$ & $\begin{array}{l}0,42 \\
0,15 \\
0,15 \\
0,04 \\
0,51 \\
0,52 \\
0,35 \\
0,40 \\
0,57 \\
0,38 \\
-0,15 \\
0,07 \\
0,29 \\
0,04 \\
0,65 \\
0,34 \\
0,10 \\
0,57 \\
-0,06 \\
0,26 \\
0,35 \\
0,36 \\
0,36 \\
0,37 \\
0,62 \\
0,57 \\
0,82 \\
0,42 \\
0,04 \\
0,63 \\
0,40 \\
0,21 \\
0,18 \\
0,29 \\
0,16\end{array}$ \\
\hline
\end{tabular}


Quadro 6 - Distribuição das notas ponderadas e a diferença de notas obtidas, em relação ao conhecimento adquirido da TPMU, segundo as etapas do estudo. São Paulo, 1994.

\begin{tabular}{|c|c|c|c|}
\hline Ficha & $\begin{array}{l}\text { Nota ponderada } \\
\text { la. etapa }\end{array}$ & $\begin{array}{l}\text { Nota ponderada } \\
\text { 2a. elapa }\end{array}$ & Diferença de nota Execução \\
\hline $\begin{array}{l}1 \\
2 \\
3 \\
4 \\
5 \\
6 \\
7 \\
8 \\
9 \\
10 \\
11 \\
12 \\
13 \\
14 \\
15 \\
16 \\
17 \\
18 \\
19 \\
20 \\
21 \\
22 \\
23 \\
24 \\
25 \\
26 \\
27 \\
28 \\
29 \\
30 \\
31 \\
32 \\
33 \\
34 \\
35\end{array}$ & $\begin{array}{l}0,16 \\
0,32 \\
0,16 \\
0,29 \\
0,21 \\
0,26 \\
0,16 \\
0,62 \\
0,93 \\
0,27 \\
0,70 \\
0,81 \\
0,44 \\
0,26 \\
0,38 \\
0,53 \\
0,66 \\
0,73 \\
0,86 \\
0,40 \\
0,44 \\
0,93 \\
0,52 \\
0,50 \\
0,63 \\
0,50 \\
0,13 \\
0,35 \\
0,47 \\
0,49 \\
0,28 \\
0,60 \\
0,34 \\
0,43 \\
0,64\end{array}$ & $\begin{array}{l}0,74 \\
0,68 \\
0,93 \\
0,88 \\
0,82 \\
0,86 \\
0,63 \\
0,65 \\
0,81 \\
0,55 \\
0,83 \\
0,78 \\
0,54 \\
0,66 \\
0,56 \\
1,00 \\
0,87 \\
0,70 \\
0,86 \\
0,60 \\
0,88 \\
0,88 \\
0,88 \\
0,77 \\
1,00 \\
1,00 \\
1,00 \\
0,81 \\
0,56 \\
1,00 \\
0,81 \\
0,75 \\
0,79 \\
0,72 \\
1,00\end{array}$ & $\begin{array}{l}0,58 \\
0,36 \\
0,78 \\
0,59 \\
0,62 \\
0,60 \\
0,48 \\
0,03 \\
-0,12 \\
0,28 \\
0,13 \\
-0,03 \\
0,09 \\
0,41 \\
0,18 \\
0,47 \\
0,21 \\
-0,03 \\
0,00 \\
0,20 \\
0,44 \\
-0,05 \\
0,36 \\
0,27 \\
0,37 \\
0,50 \\
0,87 \\
0,45 \\
0,09 \\
0,51 \\
0,53 \\
0,15 \\
0,46 \\
0,29 \\
0,36\end{array}$ \\
\hline
\end{tabular}

$O$ teste t-pareado foi obtido calculando se as notas ponderadas nas duas etapas e as diferenças de notas de cada estudante (quadro 5 e 6), a seguir, calculou-se a média das diferenças das notas e o desvio-padrão onde se obteve o " $t$ ", o " $t$ " para habilidade foi $t=8,70$; e $t=7,89$ para o conhecimento adquirido. Os testes revelaram diferenças entre as duas etapas, com nível de significância de $5 \%$, indicando que a prática foi efetiva para o aprendizado dessa técnica, visto que a média de notas foi maior na $2 a$. etapa quando comparada com a 1a. etapa.

Face a estes dados, pode-se reafirmar, através da análise global, que a experiência prática foi determinante para aquisição da habilidade, uma vez que os estudantes executaram a técnica melhorando o seu desempenho, reduzindo os erros e aproximando-se do modelo padrão. 


\section{4 - CONCLUSÃo}

Este estudo permitiu-nos concluir, atraves do teste t-pareado, $t=8,70$ (habilidade), e $t=7,89$ (conhecimento), que ocorreram diferenças significativas no desempenho dos estudantes aposs experiência prática, demonstrando a importância da prática no processo de ensino-aprendizagem de habilidades predominantemente perceptivo-motora como a TPMU.

MURAMATSU, C.H.; MIYADAHIRA. A.M.K. The teaching-learning process of the uterine measurement and palpation techinique: the influence practice. Rev.Esc.Enf.USP, v.30, n.3, p.456-72, dec. 1996.

The present study related to the teaching-learning process concerning the uterine measurement and palpation technique, it was intended to analyze the influence practice had on the Undergraduation Nursing students' skills acquired knowledge by the use of that technique. Applying a standard instrument previously developed for this study, the students evaluation was carried out in two phases. The results showed a significant improvement in the students' performance skills and descriptive knowledge. Thus, this study was able to stress the importance of practice for the motor-perceptive skills in the teaching-learning process.

UNITERMS: Motor-perceptive skill. Palpation and height uterine measurement.

\section{5 - ANEXOS}

\section{ANEXO I}

TÉCNICA DE PAL PAÇÃo E MENSURAÇÃo U'TEIRINA

Nome do aluno:

Idade: Grupo. Etapa

Data

Tempo: Início. Término:

Número de mensurações efetuadas

1- Lavar as mãos.

2- Orientar a puérpera.

3- Pedir para esvaziar a bexiga

4- Posicionar a puérpera adequadamente.

5- Colocar-se ao lado da puérpera..

6- Aquecer as mãos.

7- Identificar a borda superior da sinfise púbica......

8- Identificar o fundo uterino.

9- Mensurar a altura uterina.

A B C

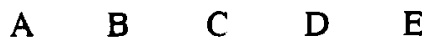

A B

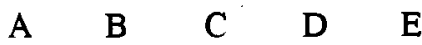

A $B$

A B

A B

A B

A B 
10- Verificar a consistência uterina.

11- Verificar a sensibilidade uterina

12- Anotar
A $\quad$ B

A $B$

A $\quad$ B $\quad$ C $\quad$ D

\section{ANEXO II}

FICHA PARA DESCRIÇÃO DA TÉCNICA DE PALPAÇÃo E MENSURAÇÃo UTERINA.

Nome do aluno. Etapa.

Descreva em itens os passos da técnica de palpação e mensuração uterina.

\section{ANEXO III}

CRITÉrios aDOTADOS PARA A AVALIAÇÃo DA APRENDIZAGEM NA TÉCNICA DE PALPAÇÃO E MENSURAḈ̃O UTERINA

\section{1 - LAVAR AS MÃOS}

A - Lavou as mãos, conforme os padrões preconizados. $(1,0)$

B - Não lavou as mãos ou lavou incorretamente. (0)

C - Lavou, mas omitiu alguns passos do item. (0.5)

2 - ORIENTAR A PUERPERA

A - Orientou explicando sobre o que é, como se realiza, a importáncia da mensuração e solicitou a sua cooperação. $(1,0)$

B - Não orientou ou orientou incorretamente. (0)

C - Orientou, mas omitiu um passo do item. $(0,75)$

D - Orientou, mas omitiu dois passos do item. (0.5)

E - Orientou, mas omitiu três passos do item. $(0,25)$

3 - PEDIR PARA ESVAZIAR A BeXIGA

A - Pediu para esvaziar a bexiga. (1.0)

B - Não pediu. (0)

\section{4 - POSICIONAR A PUERPERA ADEQUADAMENTE}

A - Posicionou a puérpera no leito, em decúbito dorsal, MMSS ao longo do tronco, MMII em extensão, abdome descoberto da apêndice xifóide à sínfise púbica. $(1,0)$

B - Não posicionou ou posicionou incorretamente. (0)

C - Posicionou, mas omitiu um passo do item. $(0,75)$

D - Posicionou, mas omitiu dois passos do item. $(0,5)$

E - Posicionou, mas omitiu três passos do item. $(0,25)$

5 - COLOCAR-SE AO LADO DA PUÉRPERA

A - Colocou-se corretamente. $(1,0)$

B - Não se colocou. (0) 
6 - AQUECER AS MÃOS

A - Aqueceu as mãos. (1.0)

B - Não aqueceu. (0)

7 - IDENTIFICAR A BORDA SUPERIOR DA SÍNFISE PÚBICA

A - Identificou corretamente. $(1,0)$

B - Não identificou. (0)

8 - IDENTIFICAR O FUNDO UTERINO

A - Identificou corretamente. $(1,0)$

B - Não identificou. (0)

9 - MENSURAR A ALTURA UTERINA

A - Mensurou corretamente. (1.0)

B - Não mensurou. (0)

10 - VERIFICAR A CONSISTÊNCIA UTERINA

A - Verificou a consistência. (1.0)

B - Não verificou. (0)

11 - VERIFICOU A SENSIBILIDADE UTERINA

A - Verificou a sensibilidade. $(1,0)$

B - Não verificou. (0)

12 - ANOTAR

A - Anotou o valor encontrado. a consistência e a sensibilidade. (1.0)

B - Não anotou. (0)

C - Anotou, mas omitiu um passo do item. $(0,67)$

D - Anotou. mas omitiu dois passos do item. $(0,33)$

\section{REFERÊNCIAS BIBLIOGRÁFICAS}

AMMON-GABERSON K. B. Adult learning principles: applications for preceptor programs. AORN J., v.45, n.4, p.961-3. 1987.

ANGELO, M. Vivendo uma prova de fogo: as experiências iniciais da aluna de enfermagem. São Paulo. 1989. 113p. Tese (Doutorado) - Instituto de Psicologia. Universidade de São Paulo.

ARAÚJO, J. O.; MARETTI. M. Propedéutica da gravidez: anamnese e exame físico. In: REZENDE, J. Obstetricia. 6.ed. Rio de Janeiro, Guanabara Koogan, 1991. cap. 9, p.159221.

BACHMAN, K. Using mental imagery to practice a specific psychomotor skill. J. Contin. Educ. Nurs., v.21, n.3, p.125-8, 1990.

BELIZAN, J.M. et al Diagnosis of intrauterine growth retardation by a simple clinical method: measurement of uterine height. Alm. J. Obstet. Gynecol., v. 131, n.6, p.643-6, 1978. 
BERGAMASCO. R. B. Ensino do exame físico das mamas: comparação entre dois métodos de instrução. São Paulo, 1994. 59p. Dissertaçăo (Mestrado) - Escola de Enfermagem,

Universidade de São Paulo.

BRASIL. Leis etc. Lei n 7.498 de 25 de junho de 1986. Dispre sobre a regulamentaçăo do exercício da enfermagem e dá outras providências. Diário Oficial da União. Brasília, 26 jun. 1986. Seçăo 1, p.9273-5.

CRANE, J. El examen fisico: un aspecto en la evaluación clinica de enfermeria. In: SANA, J. M.: JUDGE. R. D. Metodos para el examen fisico en la practica de enfermeria. Cali, Organización Panamericana de la Salud. 1977. cap.2. p.11-26.

DARIDO, S.C. Efeitos de dois procedimentos de apresentaçăo da informação na aprendizagem motora: demonstração e instrução verbal. São Paulo. 1991. 114p. Dissertação (Mestrado) Escola de Educação Física. Universidade de São Paulo.

DELASCIO, D.: GUARIENTO, A. Obstetrícia normal. 3.ed. São Paulo, Sarvier, 1987 cap.23, p.377-88: Puerpério.

ENGSTROM, J. L.; WORK. B. A. Pre natal prediction of small and large for gestacional age neonates. J. Obstet. Gynecol Neonatal Nurs., v.21, n.6, p.486-95, 1992.

ENGSTROM, J. L.; SITTLER. C. Fundal Height Measurements: Part 1 techniques for measuring fundal height. J. Nurse Midwifery., v.38, n.1. p.5-16, 1993.

ENGSTROM, J. L. et al. Fundal height measurement: Part 3 the effect of maternal position on fundal height measurements. J. Nurse Midwifery. v. 38, n.1, p.23-7, 1993.

ENGSTROM. J. L.: MCFARLIN, B. L.: SAMPSON, M. B. Fundal height measurement: Part 4 accuracy of clinicians identification of the uterine fundus during pregnancy. J. Nurse Midwifery. v. 38. n.6. p.318-23. 1993.

ENGSTROM, J. L.; SITTLER, C. P.; SWIFT, K. E. Fundal height measurement: Part 5 the effect of clinician bias on fundal height measurements. J. Nurse Midwifery., v. 39, n.3, p. 130-41, 1994.

FARRER, H. Maternity care. 2: ed. New York. Churchill Livingstone. 1990. cap. 20, p.205-16: Postnatal care.

FRIEDLANDER. M. R. O ensino dos procedimentos básicos no laboratório de enfermagem: comparaçáo entre dois métodos de instruçăo. Sao Paulo. 1984. 124p. Tese (Doutorado) Escola de Enfermagem, Universidade de São Paulo.

GOMEZ, G. E.; GOMEZ, E. A. The teaching of psychomotor skill in nursing. Nurse Educ. v.9, n.4, p.35-9, 1984.

HAWKINS, J. W.: GORVINE, B. Postpartum nursing: health care of women. New York, Springer, 1985. cap. 3, p. 34-47: Immediate postpartum assessment. nursing augmentation and intervention.

JENSEN, M. T. D.; BOBAK. I. M. Maternity and gynecologic care: the nurse and the family. 3.ed. Toronto, Mosby, 1985 a. cap.16.p.309-31: Prenatal period: nursing assessment and diagnosis .

JENSEN. M. T. D.; BOBAK, I. M. Maternity and gynecologic care: the nurse and the family. 3. ed. Toronto. Mosby. 1985 b. cap. 31.p. 737.55: Physiology and nursing assessment and diagnosis .

KIMURA, M. Ensino e aprendizagem do exame físico: análise do processo pelo exame das pupilas. Săo Paulo. 1991. 138p. Tese (Doutorado) - Escola de Enfermagem. Universidade de São Paulo. 
KUSHNIR, T. Stress and social facilitation: the effects of the presence of an instructor on student nurse behaviour. J. Adv. Nurs., v.11, p.13-9, 1986.

MAGILL, R. A. Aprendizagem motora: conceitos e aplicaçōes. São Paulo, Edgard Blucher, 1984 a. cap. 1. p.2-23: O domínio motor.

MAGILL, R. A. Aprendizagem motora: conceitos e aplicações. São Paulo. Edgard Blucher, 1984 b. cap. 2, p.24-52: A natureza da aprendizagem

MAGILL. R. A. Aprendizagem motora: conceitos e aplicaçסes. Sāo Paulo. Edgard Blucher, 1984 c. cap. 9, p. 194-210: Transferência da aprendizagem

MAGILL. R. A. Aprendizagem motora: conceito e aplicações. São Paulo, Edgard Blucher, 1984 d. cap. 11, p.237-61: Motivação.

MELO. J. M.C. Efeitos da prática mental em tarefas motoras de diferentes envolvimentos cognitivos. Sao Paulo. 1993. 60p. Dissertação (Mestrado) - Escola de Educaçăo Física. Universidade de São Paulo.

MIYADAHIRA, A. M. K. Processo de ensino - aprendizagem de habilidades psicomotoras: análise da técnica de injeção intramuscular. São Paulo, 1990, 243p. Tese (Doutorado) Escola de Enfermagem. Universidade de São Paulo.

MIYADAHIRA. A. M. K.: KOIZUMI. M. S. Medida das capacidades psicomotoras envolvidas na técnica da injeçăo intramuscular. Rev. Esc. Enf. USP. v.27.n.2. p.263-80. 1993.

NEME, B. Obstetrícia básica. São Paulo. Sarvier, 1994 a. cap.13, p.68-101: Propedéutica da obstetrícia .

NEME. B. Obstetrícia básica São Paulo. Sarvier, 1994 b. cap. 87. p.597-610: Infeç̧ão puerperal.

NOGUEIRA. A. S. Aplicação de injetáveis via intramuscular: comparação entre dois métodos de ensino. Ribeirão Preto, 1995. 154p. Tese (Doutorado) - Escola de enfermagem de Ribeirão Preto, Universidade de São Paulo.

OERMANN, M.H. Psychomotor skill development. J. Contin. Educ. Nurs. v.21, n.5, p.202-4, 1990.

PASSOS, S.C.E. Efeitos da variabilidade da prática na aprendizagem de uma habilidade motora. São Paulo, 1989. 102p. Dissertação (Mestrado) - Escola de Educação Física. Universidade de São Paulo.

REEDER, S. J.; MARTIN, L. L. Maternity nursing: family, newborn. and womens health care. 6. ed. Philadelphia, Lippincott. 1987. cap.31, p.594-615: Nursing care in the postpartum period.

ROGERS: J. Ensino de adultos. Lisboa. Martins Fontes. 1976 a. cap.2, p.35-60: O que sente o estudante adulto.

SANCHES; A. B. Estágios de desenvolvimento motor em estudantes universitários na habilidade básica de arremessar. São Paulo. 1989. 81p. Dissertação ( Mestrado) - Escola de Educação Física, Universidade de São Paulo

TANI, G. Significado, deteç̧ão e correção do erro de performance no processo ensino aprendizagem de habilidades motoras. Rev. Bras. Ci. Mov.. v.3, n.4, p.50-8. 1989.

TANI, G. et al. Educação física escolar: fundamentos de uma abordagem desenvolvimentista. São Paulo, EPU. 1988.

WORTHEN, N.: BUSTILLO, M. Effect of urinary bladder fullness on fundal height measurements. Am. J. Obstet. Gynecol. v. 138, n. 7, p.759-62, 1980. 